\title{
Solving N-queen Problem Using Genetic Algorithm by Advance Mutation Operator
}

\author{
Vinod Jain ${ }^{1}$, Jay Shankar Prasad ${ }^{2}$ \\ ${ }^{1}$ Department of CSE/IT, MVN University Palwal, India \\ ${ }^{2}$ Department of Computer and Information Science, MVN University Palwal, India
}

\section{Article Info \\ Article history: \\ Received 31 Oct, 2017 \\ Revised Jun 12, 2018 \\ Accepted Jun 30, 2018 \\ Keyword: \\ Cross over operator \\ Genetic algorithm \\ Mutation over operator \\ $\mathrm{N}$-queen problem \\ Soft computing}

\section{Corresponding Author:}

Vinod Jain,

Department of CSE/IT,

MVN University Palwal, India.

Email: jainvinod81@gmail

\begin{abstract}
$\mathrm{N}$-queen problem represents a class of constraint problems. It belongs to set of NP-Hard problems. It is applicable in many areas of science and engineering. In this paper $\mathrm{N}$-queen problem is solved using genetic algorithm. A new genetic algoerithm is proposed which uses greedy mutation operator. This new mutation operator solves the N-queen problem very quickly. The proposed algorithm is applied on some instances of $\mathrm{N}$-queen problem and results outperforms the previous findings.
\end{abstract}

Copyright () 2018 Institute of Advanced Engineering and Science. All rights reserved.

\section{INTRODUCTION}

$\mathrm{N}$-Queen problem is a problem in computer science that is not solvable using traditional algorithms. In $\mathrm{N}$-Queen problem, $\mathrm{N}$ number of queens have to be placed on a chess board of $\mathrm{N}$ rows and $\mathrm{N}$ columns. The Queens must be placed such that no two queens should clash each other. So in every row and every column the challenge is to place only one queen. In N-queen problem some constraints have to be satisfied, so $\mathrm{N}$-queen problem is also known as constraint satisfaction problem. It consists of some variables, some values to these variables and some constraints that are to be satisfied. Figure 1 shows a sample solution of 8 Queen problem. Q1,Q2,Q3 are queens placed on the chess board.

The N Queen problem has many applications in science and engineering. It can be used to solve problems in real time computer systems, error correction and detection, designing of communication systems, designing of VLSI circuits, resource management in computer systems, testing of VLSI circuits, scheduling of tasks in operating system, solving routing problems in computer networks, balancing of load on different microprocessors in computers, parallel processing in optics, controlling traffic, data and image compression, storage of memory parallel, prevention of deadlocks in operating system, assignment of tasks and many more. In recent years many researchers try to solve $\mathrm{N}$-queen problem.

$\mathrm{N}$-queen problem can be solved using backtracking algorithm. In recent years many authors are working on solution of $\mathrm{N}$-queen problem and its various applications in science and engineering [1]-[4]. In literature efforts have been made to solve $\mathrm{N}$-queen problem using metaheuristic techniques such as Genetic Algorithm GA, Ant Colony Optimization ACO, Particle Swarm Optimization PSO, Simulated Annealing SA etc. [5]-[7]. [8], [9] Vinod Jain and Jay Shankar Prasad apply genetic algorithm to solve Travelling Salesman problem and found better results for it. These efforts try to solving $\mathrm{N}$-queen problem for smaller values of $\mathrm{N}$. In [10] author improves the performance of solving N-queen problem by using multi core processors. Author 
used an OpenMP technique to solve N-queen problem. Jalal eddin Aghazadeh heris et.al [11] tries to solve $\mathrm{N}$-queen problem using a modified genetic algorithm. Author found that the improved Genetic Algorithm solve N-queen problem very quickly as compared to standard Genetic Algorithm. Yuh-Rau Wang et.al, [12] apply swarm refinement PSO(SR-PSO) to solve N-queen problem. Author suggested that the proposed SR-PSO solve the N-queen problem better as compared to existing Permutation PSO (Per-PSO) and Genetic Algorithm. [13] Author apply genetic algorithm to improve the dynamic changing environment in smart antennas. [14] Apply ACo and GA in wireless sensor network to optimize the location of controllers. [15] Author apply GA to find the cause of heart attacks. [16] Author apply GA in artificial immune system. Author [17] apply GA to overcome the problem of traffic lights. Lijo V. P. and Jasmin T. Jose [18] solves the N-queen problem by pridiction.

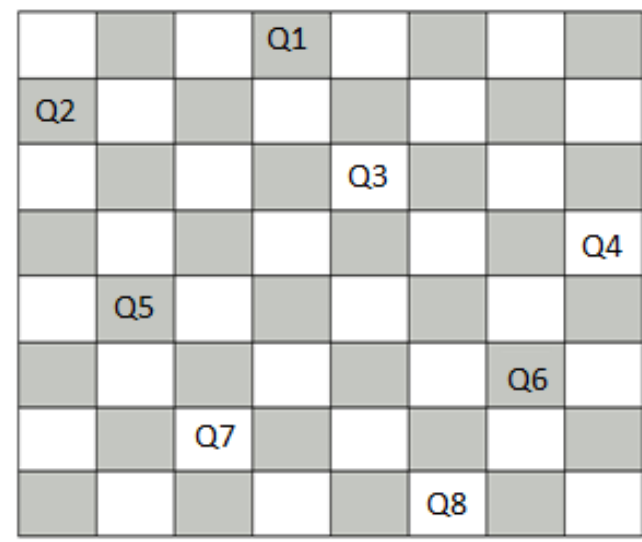

Figure 1. A sample solution of 8-Queen problem

\section{RESEARCH METHOD}

In this work new genetic algorithm is proposed to solve N-Queen problem. The proposed AGA algorithm is differing than the other Genetic Algorithm while applying its advanced mutation operation. The mutation operation does some accidental changes in the population. This step is performed after cross over operation. In this work those part of the chromosomes is mutated which are producing clashes with other queens. This the mutation operation reduces the clashes in a chromosome and improves the fitness of that chromosome. A set of newly mutated chromosomes are generated and added in the population just like the cross over operation.

Let chr-1 is a chromosome of 10-Queen problem. In the list positions of Queens in different columns (from 0-9) are given in chr-1(Chromosome-1). Chromosome before removing column clash is (Chr-1)

$\begin{array}{llllllllll}3 & 6 & 1 & 8 & 0 & 5 & 4 & 2 & 5 & 9\end{array}$

Clash Count $=10$

The number of queens which are having clashes with other queens are 10.

A queen position which is producing clashes if found and queen at that location is swapped with other queen. Queen at position 5 is replaced with 7. So the chromosome after mutation is:

$$
\begin{array}{llllllllll}
3 & 6 & 1 & 8 & 0 & 7 & 4 & 2 & 5 & 9
\end{array}
$$

Clash Count $=4$

The mutated chromosome has only 4 clashes. Thus this advance mutation operation is removing clashes very quickly and thus finding the solution in less time. The proposed genetic algorithm with Advanced Mutation Operation is as follows:

Proposed Algorithm

1. Create initial population of chromosomes

2. Find fitness of current population

3. If stopping criteria reached, then stop otherwise continue

4. Perfrom natural selection

5. Perform cross over and generate new children

6. Add newly generated children in current population

7. Find fitness of current population 
8. Sort the chromosomes by decreasing order of the fitness

9. Generate next population

10. Apply proposed mutation

11. Go to Step-3.

\section{RESULTS AND ANALYSIS}

The proposed genetic algorithm is implemented in JAVA. Results are calculated in terms of execution time of the proposed genetic algorithm to solve different instances of $\mathrm{N}$-queen problem. The algorithm is applied on two instances of $\mathrm{N}$-queen problem having 8 and 50 queens. The obtained results are shown in Table 1. The results are compared with other algorithm to solve $\mathrm{N}$-queen problem. The table shows that the results obtained by this proposed algorithm are better for almost all the instances of $\mathrm{N}$-queen problem.

Table 1. Comparison of Results

\begin{tabular}{|c|c|c|c|c|c|c|}
\hline Queens/Algorihtm & $\begin{array}{c}8 \\
\text { Time }(\mathrm{sec})\end{array}$ & $\begin{array}{c}16 \\
\text { Time }(\mathrm{sec})\end{array}$ & $\begin{array}{c}30 \\
\text { Time }(\mathrm{sec})\end{array}$ & $\begin{array}{c}40 \\
\text { Time }(\mathrm{sec})\end{array}$ & $\begin{array}{c}50 \\
\text { Time }(\mathrm{sec})\end{array}$ & $\begin{array}{c}100 \\
\text { Time }(\mathrm{sec})\end{array}$ \\
\hline SRPSO & - & - & 6.59 & 23.73 & 40.12 & - \\
\hline Per-PSO & - & - & 10.32 & 34.30 & 53.25 & - \\
\hline Old-GA & - & - & 17.29 & 35.66 & 54.43 & - \\
\hline New Proposed GA & 0.0307 & 0.0972 & 0.3420 & 0.7885 & 1.0443 & 14.3688 \\
\hline
\end{tabular}

Figure 2 shows a graph representation of performance of different existing algorithm and proposed genetic algorithm to solve N-queen problem. Graph is showing the results for 8,16,30,40, 50 and 100 Queens. These are the N-queens instances for which results are shown in the paper. Graph shows that the proposed algorithm obtained results in less time as compared to other existing algorithm. It can be concluded from the results that the proposed genetic algorithm is finding the solution for given instances of $\mathrm{N}$-queen problem in minimum time. Results are better than the best existing algorithms (SRPSO).

\section{Results for 30 Queens, 40 Queens and 50 Queens}

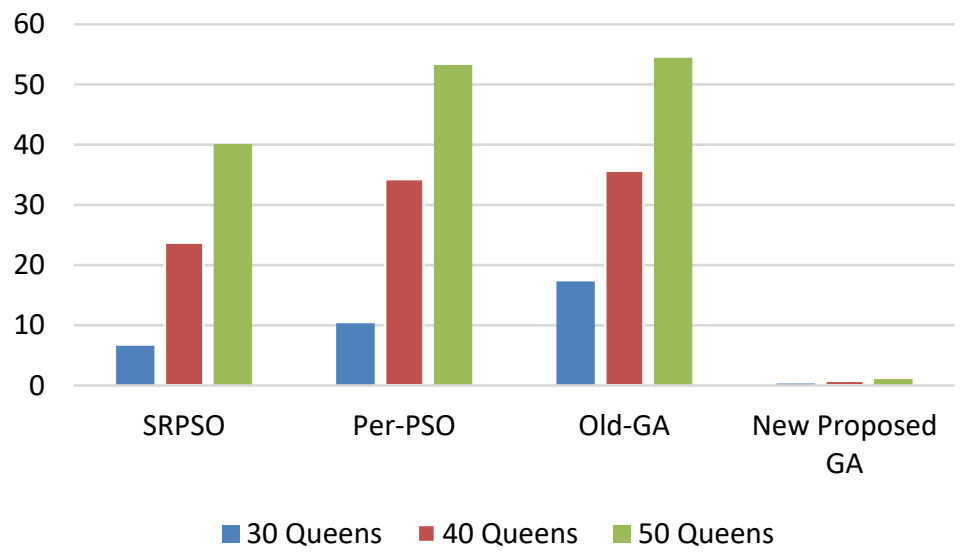

Figure 2. Graph showing comparison of results

Table 2 shows results of solving 13-Queen and 14-Queen problem using proposed algorithm. Results are compared with the results of [18] which also solves the13-Queen and 14-Queen problem. The proposed algorithm is taking very less time and less number of iterations as compared to solution found by Lijo V. P. and Jasmin T. Jose [18]. Figure 3 shows snapshot showing solution of 13-queen and 14-queen problem. 
Table 2. Comparison of Results

\begin{tabular}{lcccc}
\hline Queens/Algorihtm & $\begin{array}{c}\text { 13-Queens } \\
\text { Time }(\mathrm{sec})\end{array}$ & $\begin{array}{c}\text { 14-Queens } \\
\text { Time }(\mathrm{sec})\end{array}$ & $\begin{array}{c}\text { 13-Queens } \\
\text { Iterations }\end{array}$ & $\begin{array}{c}\text { 14-Queens } \\
\text { Iterations }\end{array}$ \\
\hline Lijo V. P. and Jasmin T. Jose [18] & 44.14 & 87.56 & $84,034,432$ & $543,672,172$ \\
New Proposed GA & 0.0492 & 0.0527 & 18 & 34 \\
\hline
\end{tabular}

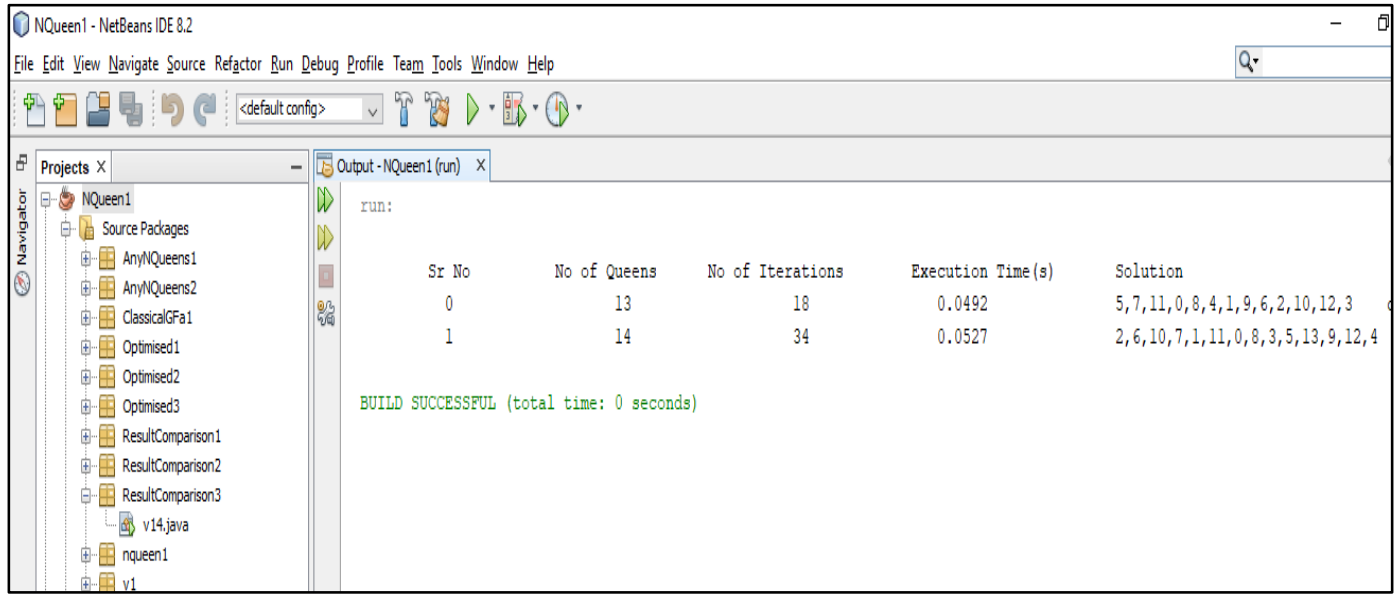

Figure 3. Snapshot showing solution of 13-Queen and 14-Queen problem

\section{CONCLUSION AND FUTURE SCOPE}

$\mathrm{N}$-queen problem can be solved using genetic algorithm. The proposed genetic algorithm uses a fast mutation operator that solves many instances of $\mathrm{N}$-queen problem in quick time. So the proposed genetic algorithm is better than the other existing algorithms. In future the proposed algorithm can be applied to $\mathrm{N}$-queen problem has large number of Queens (i.e. No of queens $>500$ ). The proposed algorithm can be further optimized to produce results more quickly. In future other genetic operators such as selection and cross over can be modified to solve the problem faster.

\section{ACKNOWLEDGEMENTS}

This work is supported by Dr. Rajeev Ratan Arora and by Dr. Sachin Gupta, Professor at MVN University palwal.

\section{REFERENCES}

[1] J. Bell, B. Stevens, "A survey of known results and research areas for s", Discrete Mathematics 309 (2009), pp. 131

[2] D. Du, J. Gu, P.M. Pardalos, Satisfiability Problem: Theory and Applications, in Proceedings of a DIMACS Workshop, vol. 35, March 11-13, 1996

[3] X. Hu, R.C. Eberhart, Y. Shi, Swarm Intelligence for Permutation Optimization: A Case Study of n-Queens Problem, in Swarm Intelligence Symposium, 2003. SIS '03. Proceedings of the 2003 IEEE. pp. 243-246, 24-26 April 2003, ISBN: 0-7803-7914-4.

[4] T. Hulubei, B. O'Sullivan, Optimal Refutations for Constraint Satisfaction Problems, in IJCAI-05, Proceedings of the Nineteenth International Joint Conference on Artificial Intelligence, Edinburgh, Scotland, UK: Professional Book Center, 2005, pp. 163-168, ISBN: 0938075934.

[5] S. Khan, Solution of n-Queen Problem Using ACO, in Multitopic Conference, 2009. INMIC 2009. IEEE 13th International, pp. 1-5, Islamabad, 14-15 Dec. 2009, ISBN: 978-1-4244-4872-2.

[6] I. Martinjak, M. Golub, Comparison of Heuristic Algorithms for the N-Queen Problem, in Proceedings of the ITI 2007 29th Int. Conf. on Information Technology Interfaces, June 25-28, 2007, Cavtat, Croatia, pp. 759-764.

[7] V.M. Saffarzadeh, P. Jafarzadech, M. Mazloom, A Hybrid Approach Using Particle Swarm Optimization and Simulated Annealing for N-queen Problem, in World Academy of Science, Engineering \& Technology; July 2010, Vol. 67, pp. 974-978.

[8] Vinod Jain, Jay Shankar Prasad,“An Optimized Algorithm for Solving Travelling Salesman Problem Using Greedy Cross Over Operator", published in 10th INDIACom; INDIACom-2016; IEEE Conference ID: 374652016 3rd International Conference on "Computing for Sustainable Global Development", 16th - 18th March, 2016 Bharati Vidyapeeth's Institute of Computer Applications and Management (BVICAM), New Delhi (INDIA) 
[9] Vinod Jain, Jay Shankar Prasad, "Solving Travelling Salesman Problem Using Greedy Genetic Algorithm GGA" , International Journal of Engineering and Technology (IJET), DOI: 10.21817/ijet/2017/v9i2/170902188 Vol 9 No 2 Apr-May 2017.

[10] A. Ayala, H. Osman, D. Shapiro, J.-M. Desmarais, J. Parri, M. Bolic and V. Groza, "Accelerating N-Queens Problem using OpenMP", 6th IEEE International Symposium on Applied Computational Intelligence and Informatics, May 19-21, 2011 Timişoara, Romania

[11] Jalal eddin Aghazadeh heris and Mohammadreza Asgari Oskoei, "Modified Genetic Algorithm for Solving nQueens Problem", 978-1-4799-3351-8/14/\$31.00 @2014 IEEE

[12] Yuh-Rau Wang, Hsieh-Liang Lin and Ling Yang,"Swarm refinement PSO for solving N-queens problem", 2012 Third International Conference on Innovations in Bio-Inspired Computing and Applications, 978-0-7695-4837$1 / 12 \$ 26.00$ @ 2012 IEEE DOI 10.1109/IBICA.2012.43

[13] Jafar Ramadhan Mohammed, "Comparative Performance Investigations of Stochastic and Genetic Algorithms Under Fast Dynamically Changing Environment in Smart Antennas", International Journal of Electrical and Computer Engineering (IJECE), Vol. 2, No. 1, February 2012, pp. 98-105

[14] Dac-Nhuong Le, "GA and ACO Algorithms Applied to Optimizing Location of Controllers in Wireless Networks", International Journal of Electrical and Computer Engineering (IJECE), Vol. 3, No. 2, April 2013, pp. 221-229

[15] Asraa Abdullah Hussein, "Improve The Performance of K-means by using Genetic Algorithm for Classification Heart Attack“, International Journal of Electrical and Computer Engineering (IJECE), Vol. 8, No. 2, April 2018, pp. 1256-1261

[16] Pongsarun Boonyopakorn and Phayung Meesad, "The Evaluated Measurement of a Combined Genetic Algorithm and Artificial Immune System”, International Journal of Electrical and Computer Engineering (IJECE) Vol. 7, No. 4, August 2017, pp. 2071-2084

[17] I Gede Pasek Suta Wijaya, Keeichi Uchimura, and Gou Koutaki, "Traffic Light Signal Parameters Optimization Using Modification of Multielement Genetic Algorithm", International Journal of Electrical and Computer Engineering (IJECE), Vol. 8, No. 1, February 2018, pp. 246-253

[18] Lijo V. P. and Jasmin T. Jose, "Solving N-Queen Problem by Prediction", International Journal of Computer Science and Information Technologies (IJCSIT), Vol. 6 (4), 2015, 3844-3848

\section{BIOGRAPHIES OF AUTHORS}
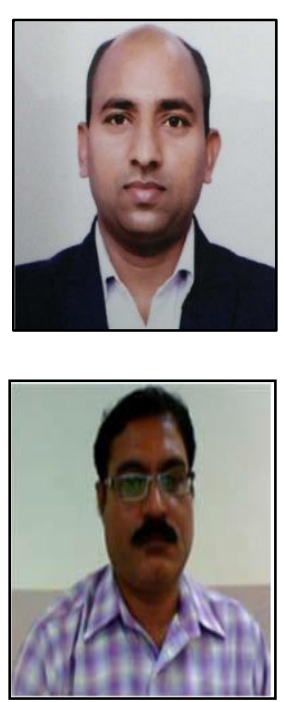

Vinod Jain Education Master of Technology(Computer Engineering) YMCA University, Faridabad, Haryana, India(2012), Master of Computer Application MCA, Kurukshetra University (2004), Research Scholar MVN University Palwal, Haryana, India. Currently working as a Assistant Professor, B.S.Anangpuria Institute of Technology and Management Faridabad, Haryana since 2008. He has published more than 8 papers international journals and international conferences. His area of research include Genetic Algorithms, NP-Complete and NP-Hard problems, Search Engine Optimization, Page ranking, Crawling, Indexing, Web mining etc. His current area of research is solving NP-Complete and MP-Hard problems using Genetic Algorithm.

Jay Shankar Prasad research interest is Artificial Intelligence, Pattern recognition, Machine learning, Computer Vision, Robotics, Humanoid Robots, Gesture Recognition, ISL Recognition, Pattern mining, Cloud computing etc .He has published Twelve papers in International journals and International conferences. He has 16 years of teaching and 3 years of software industry experience. He also guided many postgraduate and under graduate level projects 